\title{
Discrete Nonlinear Contraction Theory based Adaptive Control Strategy for a Class of Hammerstein Systems with Saturated Hysteresis
}

\author{
Zhiyong Sun ${ }^{1}$, Yu Cheng ${ }^{2}$, Ning $\mathrm{Xi}^{1}$, Congjian $\mathrm{Li}^{1}$, Sheng $\mathrm{Bi}^{1}$, Jiangcheng Chen ${ }^{1}$, and Di Guo ${ }^{1}$
}

\begin{abstract}
Smart materials actuated devices have been attracting tremendous attentions for decades, especially nowadays. Smart material actuators have been prevalently utilized in the fields such as soft robots and nano-engineering for their outstanding merits such as flexibility and ultrahigh spatial resolution. Although, usage of these smart material actuators can facilitate transmission systems design, problems show up in the control counterpart, which is still a challenging topic. As common phenomena, these actuators exhibit inherent hysteretic input-output relation which usually cause systems behave unexpectedly compared to conventional actuator such as the motors. Moreover, some actuators' behaviors can be slightly or seriously influenced by the external and internal factors, such as environmental temperature and humidity and the instinctive deterioration caused by usage. To cope with the challenging control tasks, adaptive control strategies, which are able to detect variation of actuator characteristics via estimation techniques, have been widely investigated and implemented. One of the promising generalized adaptive control schemes is developed based on the nonlinear contraction theory (NCT), which brings a more transparent and simpler process in controller design and convergence analysis. This paper proposes to design a discrete nonlinear adaptive control strategy based on the Discrete NCT (DNCT) to tackle a class of general hysteretic system considering saturation and time varying properties. The proposed control strategy combines feedback control law, adaptive law and the hysteresis compensator to guarantee desired input-output dynamic relation of the closedloop system. Sufficient stability condition is given and verified through simulations, test result shows the system tracking error and parameters estimation error converge to zero exponentially fast.
\end{abstract}

\section{INTRODUCTION}

To date, smart materials such as piezoceramics [1], [2], Shape Memory Alloy (SMA) [3], [4], Ionic Polymer Metal Composite (IPMC) [5], [6], Fishing Line (FL) [7], [8] have been bringing tremendous opportunities to fields such as robotics and nano-engineering, because of their outstanding merits of pliability, ultrahigh spatial resolution. Although, employing these smart material actuators can facilitate transmission systems design, the problems show up in the control systems development, which is still challenging currently. As common phenomena, these actuators exhibit inheren$\mathrm{t}$ hysteresis and dynamics coupling characteristics which

This work was supported in part by the U. S. Army Research Laboratory and the U. S. Army Research Office under the Grant W911NF-16-1-0572.

${ }^{1}$ Z. Sun, N. Xi, S. Bi, C. Li, J. Chen and D. Guo are with Department of Industrial and Manufacturing Systems Engineering, The University of Hong Kong, Pokfulam, Hong Kong SAR \{sunzy, xining, congjian.li, shengbi, jcchen, guodi\}ahku.hk

${ }^{2} \mathrm{Y}$. Cheng is with Department of Electrical and Computer Engineering, Michigan State University, East Lansing, MI, 48824, USA chengyu 9 @msu.edu usually cause systems behave unexpectedly compared to conventional driven methods such as motors and hydraulic power. Moreover, most smart materials commonly exhibit saturated behaviors which are slightly or seriously influenced by many external and internal factors, such as variation of the environmental temperature and humidity as well as the instinctive deterioration caused by usage [6], [8]-[10].

The varying hysteretic and dynamic properties put obstacles in the way of achieving desirable actuation precision of smart material. To tackle the challenging control tasks, adaptive control strategies, which are able to detect variation of actuator characteristics via estimation techniques, have been widely investigated and implemented. For instance, an integrated Kalman observer based LQG controller was designed to reduce the hysteresis to achieve desirable inputoutput dynamic behavior of piezoceramics driven nanopositioner [11]; a Prandtl-Ishilinskii (PI) hysteresis compensator based sliding-mode-like adaptive controller was developed to regulate the time varying hysteretic and dynamic behavior of IPMC [6]; a Preisach type hysteresis model based adaptive controller with guaranteed tracking error boundary was proposed to adaptively tune parameters to properly regulate the piezo-positioner according to various circumstances [12]. Although, expected control performances have been achieved, design of such types of advanced controllers shows great theoretical complexity at searching for effective control schemes (including control law and adaptive law), and constructing available Lyapunov type stability function to analyze convergence of the built closed-loop system.

To reduce adaptive controller design complexity, one of the promising generalized adaptive control schemes is developed based on the nonlinear contraction theory (NCT) [13], [14], which employs a "top-down" design methodology that leads to a relatively more transparent and simpler process through building a proper virtual system [15]-[17]. Different from previous studies on NCT based adaptive controllers design for continuous system, motivated by the fact that discrete control scheme is more practical for nowadays implementation, this paper focuses on developing the discrete nonlinear adaptive control strategy based on the Discrete NCT (DNCT) to tackle a class of general hysteretic system considering saturation and time varying properties. The proposed control strategy combines feedback control law, adaptive law and the hysteresis compensation technique together to guarantee desirable dynamic input-output relation of the closed-loop system.

In this research, we proposed the DNCT based adaptive controller to tackle a class of systems with saturated 
hysteretic problems containing unknown parameters, the remaining contents are arranged as follows: In section II, the system with saturated hysteretic characteristic will be formulated, and the exact inversion of the saturated hysteretic component will be introduced. Section III will first review the essentials of the DNCT and then will present the design details of the DNCT based adaptive controller, sufficient stability condition will also be derived. Section IV will verify the proposed control strategy through simulations. Conclusions are summarized in the section V.

\section{Hammerstein Systems with SATURATEd HYSTERESIS AND INVERSE COMPENSATION}

\section{A. Hammerstein Systems with Saturated Hysteresis}

Smart materials actuated devices, such as SMA driven dexterous robotic hand and piezoceramics driven nanomanipulator have been successfully implemented in many applications for their outstanding merits such as high power density and ultrahigh spatial resolution [3], [18]. The main disadvantages inhibiting smart materials becoming as popular as the conventional actuators are their inherent hysteresis and susceptibility to the external factors, such as environmental temperature, which influence the actuators' input-output relation a lot.

To represent the actuation behavior of smart materials mathematically, the hysteresis-dynamics cascade model [12], [19], [20], a type of Hammerstein model, has been widely employed in advanced controllers design. The Hammerstein model is prevalently constructed as nonlinear hysteretic component cascaded to one linear dynamic part which is commonly selected as a transfer function for easy uncertainty analysis [21]. Behavior of hysteretic part is more complicated than the linear transfer function or the conventional differentiable nonlinear function, since it cannot be linearized locally due to the memory characteristic [1]. To represent hysteresis mathematically, lots of practical modeling approaches have been reported, the prevalent including Bouc-Wen hysteresis model [2], Preisach model [22] and Prandtl-Ishlinskii (PI) model [1]. For reasons of easy compensation and analysis, the PI model and its exact inversion have been widely implemented in advanced controllers design [23], [24]. To improve modeling efficiency of saturation and asymmetric characteristics, advanced derivatives of PI type models have been developed [18], [25]-[27], and the invertible ones such as Modified PI (MPI) model [25] and Generalized PI (GPI) model [26] have been implemented a lot.

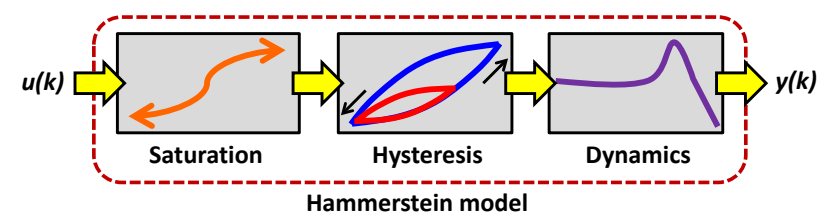

Fig. 1. Hammerstein model for smart material actuators considering saturation, hysteresis and dynamics characteristics.

To handle the case of saturated hysteresis, this research combines merits of MPI and GPI model together to build the Hammerstain model as shown in the Fig. 1, which can be mathematically represented as the discrete model (1),

$$
y(k)=D\left[u_{h}\right] \circ H\left[u_{s}\right] \circ S[u](k),
$$

where $u(k), y(k)$ is the input and output of the Hammerstain model, respectively; $S[\cdot]$, as represented in (2), denotes the saturation function which is memoryless and nonlinear; $H[\cdot]$, as shown in (3), represents the conventional PI hysteresis which is invertible; $D[\cdot]$, as shown in (4), stands for the dynamics in the discrete transfer function form; o represents the operator of composition.

$$
\begin{gathered}
u_{s}(k)=S[u](k)=\arctan \left(\theta_{1} u(k)+\theta_{0}\right), \quad \text { s.t. } \theta_{1}>0, \\
\left\{\begin{array}{c}
u_{h}(k)=H\left[u_{s}\right](k)=w_{0} u_{s}(k)+\sum_{i=1}^{i=h} w_{i} F_{r_{i}}\left[u_{s}\right](k), \\
\text { s.t. } \quad w_{0}>0, \quad w_{i} \geq 0, \\
F_{r_{i}}\left[u_{s}\right](k)=\max \left\{u_{s}(k)-r_{i},\right. \\
\left.\min \left\{u_{s}(k)+r_{i}, F_{r_{i}}\left[u_{s}\right](k-1)\right\}\right\}, \\
y(k)=D\left[u_{h}\right](k)=\frac{b_{m} z^{m}+b_{m-1} z^{m-1}+\cdots+b_{0}}{z^{n}+a_{n-1} z^{n-1}+\cdots+a_{1} z+a_{0}} u_{h}(k),
\end{array}\right.
\end{gathered}
$$

where $\left\{\theta_{0}, \theta_{1}\right\},\left\{w_{0}, \cdots, w_{h}\right\},\left\{a_{0}, \cdots, a_{n-1}\right\}$ and $\left\{b_{0}, \cdots, b_{m}\right\}$ denote model parameters (weight gains need to be identified for building the Hammerstain model); $r_{i}$ denotes the predefined $i^{t h}$ threshold of PI hysteresis part.

(a)
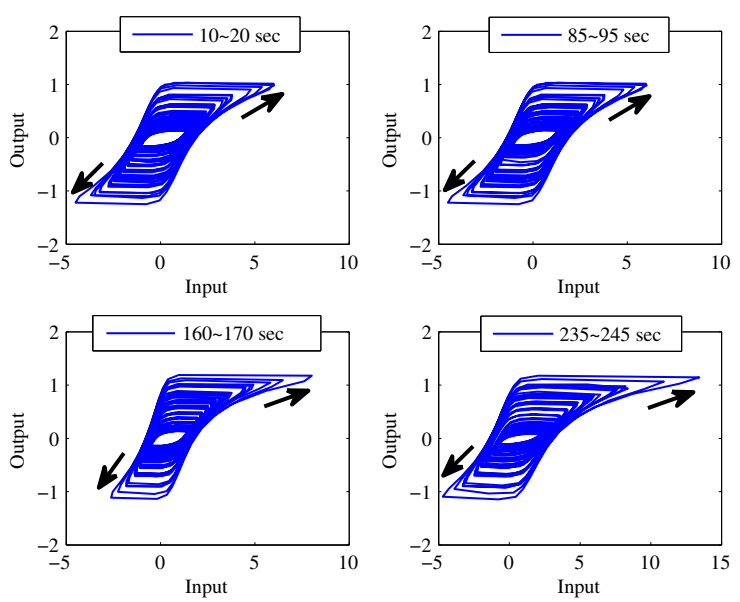

(b)

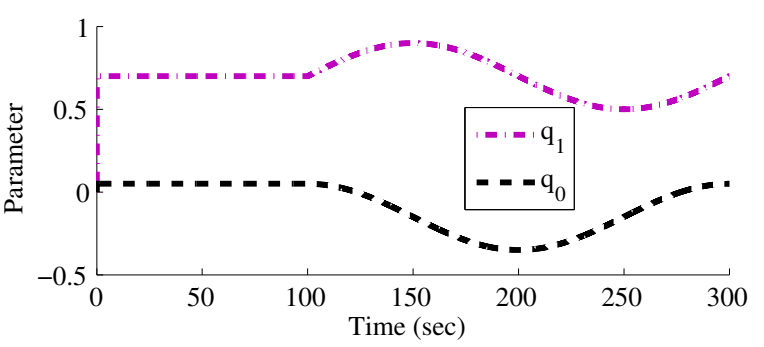

Fig. 2. Illustration of saturated hysteresis varying with parameter $\theta_{1}$ and $\theta_{0}$, (a) input-output relation of saturated hysteresis, (b) saturation parameter $\theta_{0}$ and $\theta_{1}$ vary with time.

When controlling these smart materials using control scheme with fixed structure (such as PID method), the desirable closed-loop system dynamic relation may not be 
maintained or may even become unstable [28], since these materials' actuation behaviors are susceptible to circumstance factors, which will lead to system parameters variation. To reflect the environment dependent features, as an instance, this paper treats the saturation relation (2) as the component with unknown parameters, indicating $\theta_{0}$ and $\theta_{1}$ are not precisely known when designing a control strategy. The illustration of varying saturated hysteresis portion with time is shown in Fig. 2. It is noted the hysteresis shape changes a lot when the parameter $\theta_{0}$ and $\theta_{1}$ varying.

\section{B. Exact Inversion of Saturated Hysteretic Portion}

Hysteresis is a type of instinctive nonlinear function which cannot be linearized locally. Therefore, it is desirable for many applications to reduce the hysteresis when designing advanced controllers [23], [24]. To reduce hysteresis, inverse compensation method is an effective way [25], [26]. For the statured hysteresis combined of (2) and (3), the inversion can be directly represented as (5),

$$
\left\{\begin{array}{l}
u_{i n v}(k)=\left(\tan \left[\Gamma_{C P I}^{-1}\left[y_{r}\right]\right](k)-\theta_{0}\right) / \theta_{1}, \\
\Gamma_{C P I}^{-1}\left[y_{r}\right](k)=\hat{w}_{0} y_{r}(k)+\sum_{i=1}^{h} \hat{w}_{i} F_{\hat{r}_{i}}\left[y_{r}\right](k), \\
\hat{w}_{0}=1 / w_{0}, \\
\hat{r}_{i}=w_{0} r_{i}+\sum_{j=1}^{i-1} w_{j}\left(r_{i}-r_{j}\right) \\
\hat{w}_{i}=-\frac{w_{i}}{\left(w_{0}+\sum_{j-1} i w_{j}\right)\left(w_{0}+\sum_{j-1}^{i-1} w_{j}\right)}
\end{array}\right.
$$

where $y_{r}(k)$ is the reference signal, and $u_{i n v}(k)$ denotes the output of the hysteresis compensator. The compensator (5) will be implemented as an essential part in the designed adaptive controller of the next section.

\section{DNCT BASEd AdAPtive CONTROL StRategy}

\section{A. Essential Results of the DNCT}

The DNCT was first introduced by Dr. Lohmiller and his colleagues [13], this theory provides a set of tools to analyze the exponential stability of discrete closed-loop systems in the general meaning. To derive the discrete adaptive control strategy for generalized systems, let discrete nonlinear system (6) be the plant,

$$
\left\{\begin{array}{l}
x_{k+1}=F\left(\theta_{k}, x_{k}, u_{k}\right) \\
\Phi_{k+1}=Q\left(\Phi_{k}, u_{k}\right) \\
z_{k}=\Phi_{k} x_{k}
\end{array}\right.
$$

where $F(\cdot): R^{q} \times R^{m} \rightarrow R^{n}$ stands for the nonlinear relation between $x_{k} \in R^{n}$ and $u_{k} \in R^{q}$ with respect to $\theta_{k} \in R^{m}$, here $x_{k}, u_{k}$ and $\theta_{k}$ denote the system states, inputs and parameters, respectively, at the moment $k ; \Phi_{k} \in R^{n \times n}$ represents an invertible matrix which is regulated by the function $Q: R^{q} \rightarrow$ $R^{n \times n}$, and $M_{k}=\Phi_{k}^{T} \Phi_{k}$ is called metric [13]; $z_{k}$ denotes the observation of $x_{k}$ measured with respect to the space $\Phi_{k}$.

Now, the definition of DNCT with respect to (6) can be represented as following:

Definition 1: The discrete dynamic system (6) is said to be contracting with respect to $x_{k} \in X$, if there exists a constant $\alpha$ satisfying the following condition:

$$
\left\{\begin{array}{l}
L_{k}^{T} L_{k} \leq \alpha^{2} I, \text { s.t. } 0 \leq \alpha<1, x_{k} \in X \subset R^{n}, \\
L_{k}=Q\left(\Phi_{k}, u_{k}\right) \frac{\partial F\left[\theta_{k}, x_{k}, u_{k}\right]}{\partial x_{k}} \Phi_{k}^{-1},
\end{array}\right.
$$

where $X$ is defined as the contracting set of the system (6).

Importance of one system being contracting is that the system starts from any point $x_{0}$ within $X$ will be able to converge to each other at least $\ln ^{\alpha} / T$ exponentially fast, where $T$ denotes the sampling period.

Remark 1: $\Phi_{k}$ can be regarded as a Jacobin matrix to map the system states $x_{k}$ to measurable states of some space. To simplify the problem discussed in this research, all system states $x_{k}$ are assumed to be directly measurable, and $\Phi_{k}$ can be then selected as the identity matrix $I$.

To derive the generalized discrete adaptive control scheme based on DNCT, the following lemmas will be necessary.

Lemma 1: Consider the discrete nonlinear system (8), and its generalized form or called virtual system (9) [15],

$$
\begin{gathered}
x_{k+1}=F\left(\theta_{k}, x_{k}, x_{k}, u_{k}\right), \quad x_{k} \in R^{n}, \\
x_{k+1}=F\left(\theta_{k}, x_{v, k}, x_{k}, u_{k}\right), \quad x_{v, k}, x_{k} \in R^{n} .
\end{gathered}
$$

If the virtual system is contracting with respect to virtual state $x_{v, k}$, then when a specific solution of (8) verifies a particular property, all trajectories of the virtual system (9) will be able to verify this property exponentially fast. This Lemma 1 comes directly from the Definition 1 .

Remark 2: For most system, the system parameters $\theta_{k}$ change very slowly in response to the environmental conditions variation. Therefore, $\theta_{k}$ should maintain constant within a relatively long period of time. For reducing analysis complexity, $\theta_{k}$ will be written as $\theta$ thereafter.

Lemma 2: Assume $L_{k}$ in (7) is uniformly bounded, if there exists $\Delta$ that can make (10) at any moment $m$ satisfied, then the system (6) is said to be contracting.

$$
\begin{gathered}
\prod_{k=m T}^{(m+\Delta) T} \lambda_{\max }\left(L_{k}^{T} L_{k}\right) \leq \beta^{2}, \\
\text { s.t. } 0 \leq \beta<1, \forall m \in Z^{+}, \Delta \in Z^{+} \cup\{0\} .
\end{gathered}
$$

where $\lambda_{\max }(\cdot)$ denotes the function to calculate the maximum absolute eigenvalue.

The Lemma 2 is an extension to Definition 1 suggesting that the virtual system (9) satisfying (10) starts from any initial $x_{0} \in X$ will be able to verify the same specific property that system (8) verifies at least $\ln ^{\beta} /(\Delta+1) T$ fast.

\section{B. DNCT based Adaptive Controller Design}

To derive the general adaptive controller for system (1), denote it as the general form (11),

$$
x_{a, k+1}=F\left(\theta, x_{a, k}, u_{a, k}\right), x_{a, 0} \in X \subset R^{n}
$$

where $x_{a, k} \in X$ is the measurable variable with $X$ representing the contracting domain; $\theta$ denotes the parameters to be estimated; $u_{a, k}$ is the system input and $F(\cdot)$ is the differentiable function with respect to $x_{a, k}, u_{a, k}$, and $\theta$.

Define $\varphi\left(x_{a, k}, x_{m, k}\right) \in R^{p}$ as the control objective function where $x_{a, k}, x_{m, k} \in R^{n}$ denotes the actual states generated by 
the physical system and the desirable states generated by a reference model at the moment $k$, respectively. Then, the ideal objective function and its dynamics can be defined as $\varphi_{m, k}\left(x_{a, k}, x_{m, k}\right)$ and (12) with the ideal control law $u_{m, k}=$ $U\left(\theta, x_{a, k}, x_{m, k}\right)$,

$$
\begin{aligned}
& \varphi_{m, k+1}=G\left(\theta, x_{a, k}, x_{m, k}\right)=\psi\left(\varphi_{m, k}\right), \text { with } \\
& \left(\frac{\partial \psi\left(\varphi_{m, k}\right)}{\partial \varphi_{m, k}}\right)^{T}\left(\frac{\partial \psi\left(\varphi_{m, k}\right)}{\partial \varphi_{m, k}}\right) \leq \eta^{2} I, \text { s.t. } 0 \leq \eta<1,
\end{aligned}
$$

suggesting dynamics of the objective function $\psi(\cdot)$ is contracting with respect to $\varphi_{m, k}$.

The goal of the adaptive controller design is to build a control scheme (13) with proper parameters to realize (14),

$$
\begin{gathered}
\left\{\begin{array}{l}
u_{a, k}=U\left(\hat{\theta}_{k}, x_{a, k}, x_{m, k}\right), \\
\hat{\theta}_{k+1}=\Theta\left(\hat{\theta}_{k}, x_{a, k}, x_{m, k}\right),
\end{array}\right. \\
x_{a, k} \in\left\{x_{a, k} \in R^{n} \mid \varphi_{m, k}\left(x_{a, k}, x_{m, k}\right)=0\right\} \text { as } k \rightarrow \infty,
\end{gathered}
$$

where $u_{a, k} \in R^{m}, \hat{\theta}_{k} \in R^{q}$ represents the actual control law and adaptive law, respectively.

Regulated by $u_{a, k}$ in (13), the actual dynamics of objective function $\varphi\left(x_{a, k}, x_{m, k}\right)$ can be represented as (15),

$$
\varphi_{a, k+1}=\psi\left(\varphi_{a, k}\right)+\left(F\left(\hat{\theta}_{k}, x_{a, k}, x_{m, k}\right)-F\left(\theta, x_{a, k}, x_{m, k}\right)\right)
$$

and $\hat{\theta}_{k}$ will be updated by the defined adaptive law (16),

$$
\begin{aligned}
& \hat{\theta}_{k+1}=\hat{\theta}_{k}+\Gamma_{c}\left(\hat{\theta}_{k}, x_{a, k}, \varphi_{m, k}, \varphi_{a, k}\right)+\Gamma_{o}\left(\hat{\theta}_{k}, x_{m, k}, x_{a, k}\right), \\
& \text { s.t. } \Gamma_{c}\left(\theta, x_{a, k}, \varphi_{m, k}, \varphi_{a, k}\right)=0, \Gamma_{o}\left(\theta, x_{m, k}, x_{a, k}\right)=0,
\end{aligned}
$$

where the term $\Gamma_{c}(\cdot)$ is related to the closed-loop identification procedure and the term $\Gamma_{o}(\cdot)$ is related to the open-loop procedure.

The closed-loop scheme consisted of (15) and (16) is named actual system, based on which the virtual system can be defined as (17),

$$
\left\{\begin{array}{l}
\varphi_{v, k+1}=\psi\left(\varphi_{v, k}\right)+\left(F\left(\theta_{v, k}, x_{a, k}, x_{m, k}\right)-F\left(\theta, x_{a, k}, x_{m, k}\right)\right), \\
\theta_{v, k+1}=\theta_{v, k}+\Gamma_{c}\left(\theta_{v, k}, x_{a, k}, \varphi_{m, k}, \varphi_{v, k}\right)+\Gamma_{o}\left(\theta_{v, k}, x_{m, k}, x_{a, k}\right),
\end{array}\right.
$$

where $\varphi_{v, k}$ denotes the virtual objective function, and $\theta_{v, k}$ represents the virtual system parameters.

It is noted that when we set $\varphi_{v, k}=\varphi_{m, k}$ and $\theta_{v, k}=\theta$ to the virtual system (17), it will become (12) which is the ideal dynamic system. According to Lemma 1, the virtual system (17) will be able to realize the control goal (14) based on the fact that (12) verifies (14).

To build the adaptive control scheme based on the virtual system (17), this paper selects the $\Gamma_{c}(\cdot)$ and $\Gamma_{o}(\cdot)$ for the adaptive law as following,

$$
\left\{\begin{array}{l}
\Gamma_{c}\left(\theta_{v, k}, x_{a, k}, \varphi_{m, k}, \varphi_{v, k}\right)=K_{c} \frac{\partial F\left(\theta_{v, k}, x_{a, k}\right)}{\partial \theta_{v, k}}\left(\varphi_{m, k}-\varphi_{v, k}\right), \\
\Gamma_{o}\left(\theta_{v, k}, x_{m, k}, x_{a, k}\right)=K_{o} \frac{\partial x_{a, k}\left(\theta_{v, k}\right)}{\partial \theta_{v, k}}\left(x_{m, k}-x_{a, k}\right)
\end{array}\right.
$$

where $K_{c}, K_{a}$ denotes the diagonal weight matrix to regulate convergence speed, and they need to be designed during construction of the adaptive controller.

To achieve asymptotically stable closed-loop system (17) using (18), the following theorem is given,

Theorem 1: Consider the plant (11) regulated by the control law $U(\cdot)$ and adaptive law $\Theta(\cdot)$ in (13), if the condition (19) is satisfied,

$$
\begin{gathered}
\prod_{k=m T}^{(m+\Delta) T}\left|\lambda_{\max }\left(G_{k}\right)\right| \leq \beta, \\
\text { s.t. } 0 \leq \beta<1, \forall m \in Z^{+}, \text {and } \Delta \in Z^{+} \cup\{0\}, \\
G_{k}=\left(I+\frac{\partial \Gamma_{c}(\cdot)}{\partial \theta_{v, k}}+\frac{\partial \Gamma_{o}(\cdot)}{\partial \theta_{v, k}}\right) \\
+\left(\frac{\partial F(\cdot)}{\partial \theta_{v, k}}\left(\frac{\partial \psi\left(\varphi_{v, k}\right)}{\partial \varphi_{v, k}}\right)^{-1} K_{c} \frac{\partial F(\cdot)}{\partial \theta_{v, k}}\right),
\end{gathered}
$$

then the closed-loop system will be able to converge to the objective function $\varphi_{m}(\cdot)$ at least exponentially fast with the constant $\max \left\{\ln ^{\eta} / T, \ln ^{\beta} /((\Delta+1) T)\right\}$.

Proof: By performing derivative to the virtual system (17) with respect to virtual objective function $\varphi_{v, k}$ and virtual system parameter $\theta_{v, k}$, the following (21) can be achieved,

$$
L_{k}=\left[\begin{array}{cc}
\frac{\partial \psi\left(\varphi_{v, k}\right)}{\partial \varphi_{v, k}} & \frac{\partial F\left(\theta_{v, k}, x_{a, k}\right)}{\partial \theta_{v, k}} \\
-K_{c} \frac{\partial F\left(\theta_{v, k}, x_{a, k}\right)}{\partial \theta_{v, k}} & I+\frac{\partial \Gamma_{c}(\cdot)}{\partial \theta_{v, k}}+\frac{\partial \Gamma_{o}(\cdot)}{\partial \theta_{v, k}}
\end{array}\right] .
$$

To calculate the system boundary eigenvalue, simple operation was performed to (21), and the equivalent form can be achieved as (22),

$$
L_{k}^{\prime}=\left[\begin{array}{cc}
\frac{\partial \psi\left(\varphi_{v, k}\right)}{\partial \varphi_{v, k}} & \frac{\partial F\left(\theta_{v, k}, x_{a, k}\right)}{\partial \theta_{v, k}} \\
0 & G_{k}
\end{array}\right] .
$$

Based on the statement (12), (23) which is derived from (19), and Lemma 2, the condition (24) can be achieved,

$$
\begin{gathered}
\prod_{k=m T}^{(m+\Delta) T} \lambda_{\max }\left(G_{k}^{T} G_{k}\right) \leq \prod_{k=m T}^{(m+\Delta) T}\left|\lambda_{\max }\left(G_{k}\right)\right|^{2} \leq \beta^{2}, \\
\lambda_{\max }\left(L_{k}^{T} L_{k}\right)=\lambda_{\max }\left(L_{k}^{\prime T} L_{k}^{\prime}\right) \leq \max \left\{\eta^{2(1+\Delta)}, \beta^{2}\right\},
\end{gathered}
$$

suggesting the closed-loop system will be able to converge to $\varphi_{m}(\cdot)$ exponentially fast with the constan$\mathrm{t} \max \left\{\ln ^{\eta} / T, \ln ^{\beta} /((\Delta+1) T)\right\}$.

\section{Controller Feasibility VerificAtion through SiMUlation COMPARISON}

\section{A. Setup of Simulation Tests}

During the simulation tests, the plant (1) of smart material actuators considering saturated hysteresis was employed, the parameters of the saturation part were set to be $\theta_{1}=0.7, \theta_{0}=0.05$; for the hysteresis part, 15 PI operators were determined, $r_{i}$ was segmented uniformly within its input range $[0,0.933]$, and weight gain $\left[w_{0}, \cdots, w_{14}\right]$ were set to $[0.025,0.008,0.038,0.048,0.003,0.019,0.012,0.0765,0.207$, $0.248,0.290,0.2025,0.215,0.189,0.232]$; the dynamic part parameters were set to $a_{0}=-0.5, b_{0}=1$ and the sampling rate was set to $100 \mathrm{~Hz}$. 
To design the proposed adaptive controller, as mentioned in section II, the parameters of the saturation part were assumed to be unknown, thus initial values were initialized as $\hat{\theta}_{1}=0.07, \hat{\theta}_{0}=0.01$. The PI hysteresis inverse compensator (5) was built based on the listed parameters. Subsequently, the whole hysteresis compensator was directly cascaded to the plant. For designing the ideal $\varphi_{m, k}$ dynamics, the integral control law with $K_{I}=15$ was selected. Based on the test setup, the reference model can be derived as (25),

$$
x_{m, k}=\frac{0.15}{z^{2}-1.5 z+0.65} x_{r, k},
$$

where $x_{r, k}$ denotes the input reference signal. Then, the objective function was defined as $\varphi_{m, k}=x_{a, k}-x_{m, k}$, serving as system tracking error. To achieve stable adaptive law, according to Theorem 1 when $\Delta$ was set to 0 and reference signal boundary was predefined as \pm 3.5 , then $K_{c}=$ $\operatorname{diag}\{0.3,0.3\}$ and $K_{o}=\operatorname{diag}\{0.1,0.1\}$ can be determined.

\section{B. Comparative Tests and Results Analysis}

To show performance of the proposed adaptive controller, two sets of numerical experiments were carried out. First, the plant (1) with fixed parameters was employed, and a desirable dynamic response generated by reference model (25) and tracking results are shown in Fig. 3(a) and (c), while the estimated parameters $\theta_{1}, \theta_{0}$ are shown in Fig. 3(b) and (d). The test results verified that the built closed-loop system (17) is asymptotically stable.

The second comparative test demonstrates importance of implementing adaptive controller when the plant is seriously influenced by environmental factors. During this test, parameter $\theta_{1}$ and $\theta_{0}$ were assumed to be varying slowly (as shown in Fig. 4(c)). An integral controller with hysteresis compensator and fixed $\theta_{1}=0.7, \theta_{0}=0.05$ (other setting is the same with the proposed adaptive controller) was employed as the contrast.

When the controller with fixed parameters was employed to regulate the plant with varying $\theta_{1}, \theta_{0}$, the control result is shown in Fig. 4(a), where one can see the desirable closedloop response cannot be well maintained, significant error shows up. When it comes to the proposed adaptive controller, the tracking error (as shown in Fig. 4(b)) is greatly reduced compared to that in (a), this result is attributed to the correct estimation of varying parameters $\theta_{1}, \theta_{0}$ shown in the plot (c). It should be pointed that, for the parameters varying case, sufficient condition of Theorem 1 cannot be satisfied, therefore only closed-loop stability can be guaranteed.

\section{CONCLUSION}

In this research, in order to improve the accuracy of smart material actuators with saturated hysteretic characteristic, the DNCT based adaptive control strategy aided by exact hysteresis inversion is proposed. The proposed control scheme shows the ability to adjust its parameters according to the designed reference model's response and the plant's actual output to better maintain accuracy of operation. Comparative numerical experiments were carried out to validate the (a)
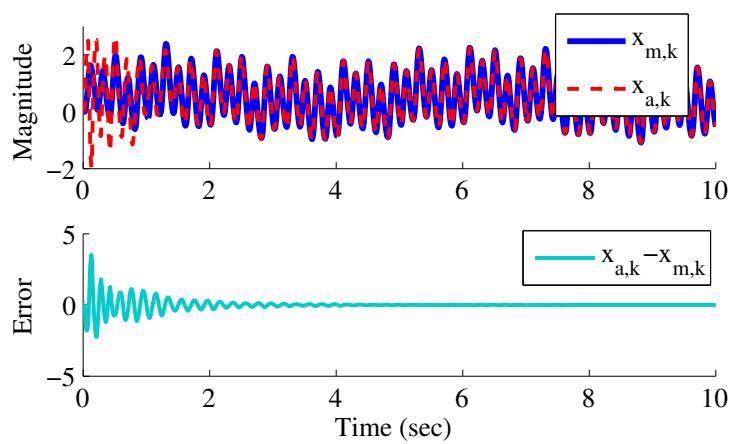

(b)

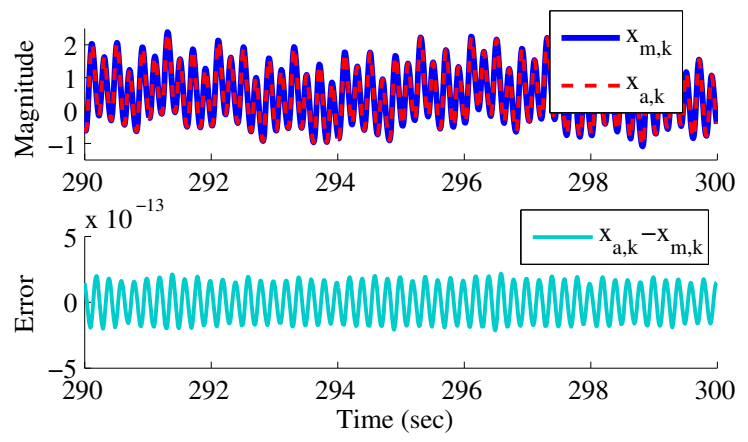

(c)

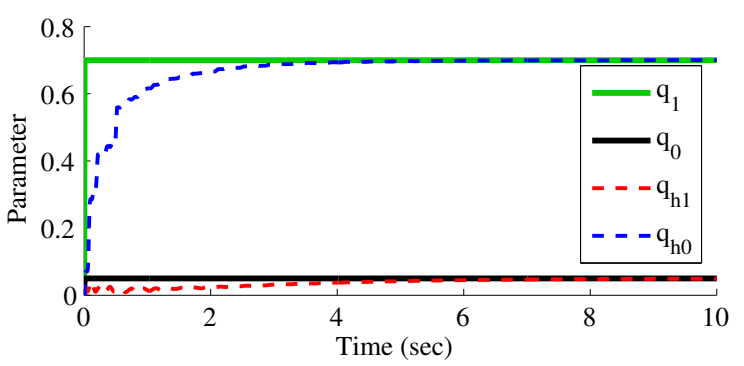

(d)

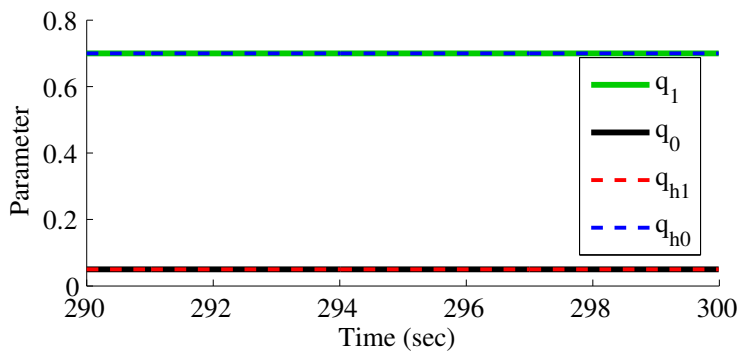

Fig. 3. Illustration of desirable dynamics tracking result when $x_{r, k}=$ $\frac{1}{2}(2+\sin (0.4 \pi k T)+\sin (2 \pi k T)+\sin (5 \pi k T)+\sin (10 \pi k T)+\sin (20 \pi k T))$ and $\theta_{1}=0.7, \theta_{0}=0.05$, (a) tracking result within 0 to 10 second period, (b) result within 290 to 300 second period, (c) parameters estimation result for the 0 to 10 second period, and (d) result for the 290 to 300 second period.

proposed control scheme. Numerical experimental results demonstrate that the proposed adaptive control scheme has a better performance compared to the controller with fixed nominal parameters when input-output relation of the plant is varying.

\section{REFERENCES}

[1] P. Krejci and K. Kuhnen, "Inverse control of systems with hysteresis and creep," IEE Proceedings-Control Theory and Applications, vol. 148, no. 3, pp. 185-192, 2001. 
(a)
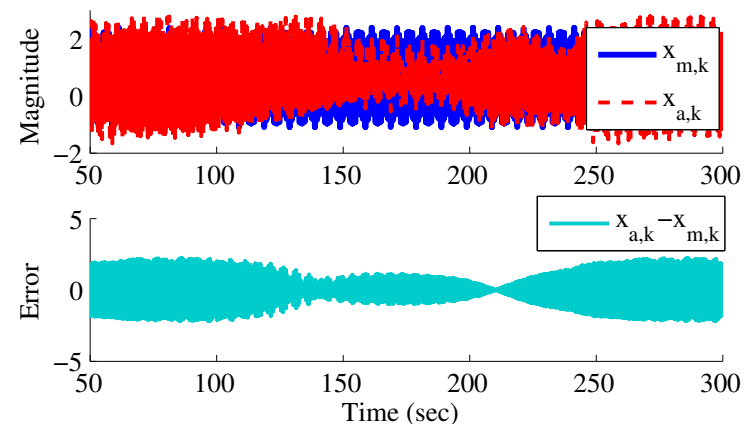

(b)
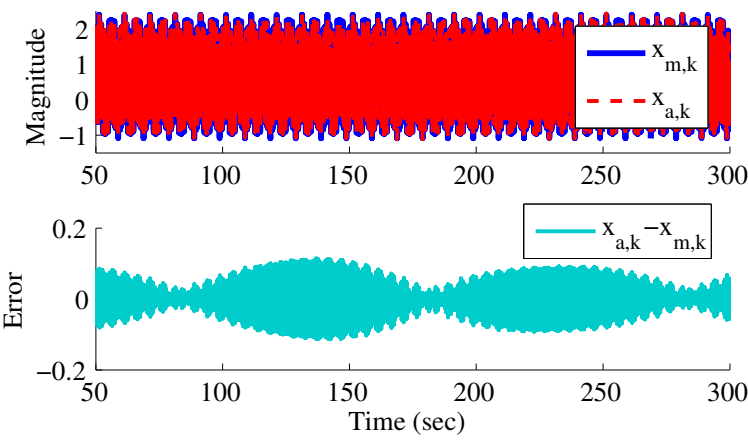

(c)

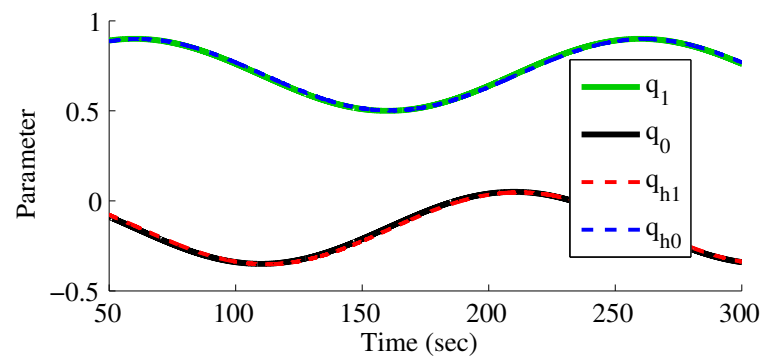

Fig. 4. Comparative test results of desirable dynamics tracking when $\theta_{1}=$ $0.7+0.2 \sin (0.01 \pi k T)$ and $\theta_{0}=-0.15+0.2 \cos (0.01 \pi k T)$, (a) control result of plant with dynamic $\theta_{1}, \theta_{0}$ using controller with nominal $\theta_{1}, \theta_{0}$, (b) result of plant with dynamic $\theta_{1}, \theta_{0}$ regulated by the proposed adaptive controller, and (c) estimation result of $\theta_{1}, \theta_{0}$.

[2] Y. Li and Q. Xu, "Adaptive sliding mode control with perturbation estimation and pid sliding surface for motion tracking of a piezo-driven micromanipulator," Control Systems Technology, IEEE Transactions on, vol. 18, no. 4, pp. 798-810, 2010.

[3] J.-S. Koh and K.-J. Cho, "Omega-shaped inchworm-inspired crawling robot with large-index-and-pitch (lip) sma spring actuators," IEEE/ASME Transactions On Mechatronics, vol. 18, no. 2, pp. 419429, 2013

[4] M. R. Zakerzadeh and H. Sayyaadi, "Precise position control of shape memory alloy actuator using inverse hysteresis model and model reference adaptive control system," Mechatronics, vol. 23, no. 8, pp. 1150-1162, 2013.

[5] H. Lei, M. A. Sharif, and X. Tan, "Dynamics of omnidirectional ipmc sensor: Experimental characterization and physical modeling," IEEE/ASME Transactions on Mechatronics, vol. 21, no. 2, pp. 601$612,2016$.

[6] Z. Sun, L. Hao, W. Chen, Z. Li, and L. Liu, "A novel discrete adaptive sliding-mode-like control method for ionic polymer-metal composite manipulators," Smart Materials and Structures, vol. 22, no. 9, p. 095027, 2013.

[7] C. S. Haines, M. D. Lima, N. Li, G. M. Spinks, J. Foroughi, J. D. Madden, S. H. Kim, S. Fang, M. J. de Andrade, F. Göktepe et al., "Artificial muscles from fishing line and sewing thread," science, vol. 343, no. 6173, pp. 868-872, 2014.

[8] J. Zhang, K. Iyer, A. Simeonov, and M. C. Yip, "Modeling and inverse compensation of hysteresis in supercoiled polymer artificial muscles,"
IEEE Robotics and Automation Letters, vol. 2, no. 2, pp. 773-780, 2017.

[9] T. Saburi, T. Tatsumi, and S. Nenno, "Effects of heat treatment on mechanical behavior of ti-ni alloys," Le Journal de Physique Colloques, vol. 43, no. C4, pp. C4-261, 1982.

[10] Z. Li, X. Zhang, C.-Y. Su, and T. Chai, "Nonlinear control of systems preceded by preisach hysteresis description: A prescribed adaptive control approach," IEEE Transactions on Control Systems Technology, vol. 24, no. 2, pp. 451-460, 2016.

[11] P.-L. Yen, M.-T. Yan, and Y. Chen, "Hysteresis compensation and adaptive controller design for a piezoceramic actuator system in atomic force microscopy," Asian Journal of Control, vol. 14, no. 4, pp. 1012 1027,2012

[12] Z. Li, X. Zhang, G.-Y. Gu, X. Chen, and C.-Y. Su, "A comprehensive dynamic model for magnetostrictive actuators considering different input frequencies with mechanical loads," IEEE Transactions on Industrial Informatics, vol. 12, no. 3, pp. 980-990, 2016.

[13] W. Lohmiller and J.-J. E. Slotine, "On contraction analysis for nonlinear systems," Automatica, vol. 34, no. 6, pp. 683-696, 1998.

[14] Q.-C. Pham, N. Tabareau, and J.-J. Slotine, "A contraction theory approach to stochastic incremental stability," IEEE Transactions on Automatic Control, vol. 54, no. 4, pp. 816-820, 2009.

[15] A. Flores-Perez, I. Grave, and Y. Tang, "Contraction based adaptive control for a class of nonlinearly parameterized systems," in American Control Conference (ACC), 2013. IEEE, 2013, pp. 2649-2654.

[16] _ - "Identification of nonlinear systems with nonlinear parameterization," in Control Conference (ECC), 2015 European. IEEE, 2015, pp. 610-615.

[17] I. Grave and Y. Tang, "A new observer for perspective vision systems under noisy measurements," IEEE Transactions on Automatic Control, vol. 60, no. 2, pp. 503-508, 2015.

[18] Z. Sun, B. Song, N. Xi, R. Yang, L. Hao, Y. Yang, and L. Chen, "Asymmetric hysteresis modeling and compensation approach for nanomanipulation system motion control considering working-range effect," IEEE Transactions on Industrial Electronics, vol. 64, no. 7, p. $5513,2017$.

[19] A. Esbrook, X. Tan, and H. K. Khalil, "Control of systems with hysteresis via servocompensation and its application to nanopositioning," Control Systems Technology, IEEE Transactions on, vol. 21, no. 3, pp. 725-738, 2013.

[20] M. Al Janaideh and D. S. Bernstein, "Adaptive control of hammerstein systems with unknown prandtl-ishlinskii hysteresis," Proceedings of the Institution of Mechanical Engineers, Part I: Journal of Systems and Control Engineering, vol. 229, no. 2, pp. 149-157, 2015.

[21] Z. Sun, L. Hao, B. Song, R. Yang, R. Cao, and Y. Cheng, "Periodic reference tracking control approach for smart material actuators with complex hysteretic characteristics," Smart Materials and Structures, vol. 25, no. 10, p. 105029, 2016.

[22] C. Natale, F. Velardi, and C. Visone, "Identification and compensation of preisach hysteresis models for magnetostrictive actuators," Physica B: Condensed Matter, vol. 306, no. 1, pp. 161-165, 2001.

[23] S. Liu, C.-Y. Su, and Z. Li, "Robust adaptive inverse control of a class of nonlinear systems with prandtl-ishlinskii hysteresis model," IEEE Transactions on Automatic Control, vol. 59, no. 8, pp. 2170-2175, 2014

[24] X. Zhang, Z. Li, C.-Y. Su, Y. Lin, and Y. Fu, "Implementable adaptive inverse control of hysteretic systems via output feedback with application to piezoelectric positioning stages," IEEE Transactions on Industrial Electronics, vol. 63, no. 9, pp. 5733-5743, 2016.

[25] K. Kuhnen, "Modeling, identification and compensation of complex hysteretic nonlinearities: A modified prandtl-ishlinskii approach," European journal of control, vol. 9, no. 4, pp. 407-418, 2003.

[26] M. Al Janaideh, S. Rakheja, and C.-Y. Su, "An analytical generalized prandtl-ishlinskii model inversion for hysteresis compensation in micropositioning control," Mechatronics, IEEE/ASME Transactions on, vol. 16, no. 4, pp. 734-744, 2011.

[27] G.-Y. Gu, C.-X. Li, L.-M. Zhu, and C.-Y. Su, "Modeling and identification of piezoelectric-actuated stages cascading hysteresis nonlinearity with linear dynamics," IEEE/ASME Transactions on Mechatronics, vol. 21, no. 3, pp. 1792-1797, 2016.

[28] C.-Y. Su, Q. Wang, X. Chen, and S. Rakheja, "Adaptive variable structure control of a class of nonlinear systems with unknown prandtlishlinskii hysteresis," IEEE Transactions on automatic control, vol. 50, no. 12, pp. 2069-2074, 2005. 